\title{
Responsabilità per inadempimento del gestore professionale e quantificazione del danno risarcibile nel contratto di gestione di portafogli
}

\author{
Responsabilidade por inadimplemento do gestor profissional e quantificação do \\ dano ressarcível no contrato de gestão de carteiras
}

\section{Civil liability for nonperformance by the professional manager and quantification of the damage in the portfolio management contract}

Monica Maria Caterina Cossu*

\begin{abstract}
Il contratto di gestione di portafogli di investimento é uno schema negoziale tipico. In caso di cattivo esito della gestione, qualora il cliente-investitore promuova causa di risarcimento per inadempimento contrattuale, l'esame del giudice deve accertare la sussistenza del danno emergente, cioé quantificare le perdite, e il lucro cessante o mancato guadagno. L'approccio della giurisprudenza appare meno soddisfacente per l'aspetto della quantificazione del lucro cessante, che spesso é drasticamente legata a parametri monetari. Sul piano delle tecniche probatorie, sono state trascurate finora le potenzialità del benchmark, o parametro oggettivo di riferimento, che pure è assurto al rango di criterio normativo.
\end{abstract}

Parole Chiave: Contratto di gestione di portafogli. Inadempimento. Responsabilità contrattuale. Danno emergente. Lucro cessante. Benchmark.

\section{Resumo}

O contrato de gestão de carteiras de investimento é um esquema negocial típico. Em caso de insucesso da gestão, se o cliente-investidor propuser ação de ressarcimento por inadimplemento contratual, o exame do juiz deve aferir a subsistência do dano emergente, isto é, quantificar as perdas e o lucro cessante, ou ganho perdido. A abordagem da jurisprudência parece menos satisfatória para o aspecto da quantificação do lucro cessante, que com frequência é relegado drasticamente a parâmetros monetários. No plano das técnicas probatórias, foram ignoradas até o momento as potencialidades do benchmark, ou parâmetro objetivo de referência, que, no entanto, foi elevado a critério normativo.

Palavras-chave: Contrato de gestão de carteiras. Inadimplemento. Responsabilidade contratual. Dano emergente. Lucro cessante. Benchmark.

\section{Abstract}

The management of investment portfolios contract is a typical contractual scheme. In case of unsuccessful management, should the custumer-investor file a damages lawsuit for the contractual nonperformance, the judge must investigate the existence of emerging damages, as well as of ceasing profits. The jurisprudence's approach seems less satisfying in what concerns the quantifying of ceasign profits, which is frequently restricted to drastic monetary paramethers. In the field of proof techniques, the potential of bechmarks, objective paramethers of reference, has been underappreciated; benchmarks, howerver, have become normative criteria.

Keywords: Management of investment portfolios. Nonperformance. Contractual liability. Emerging damages. Ceasing profits. Benchmark.

Dottore. Professore associato dell'Università di Sassari Dipartimento di Scienze Economiche e aziendali - DiSEA. Sassari - Itália. E-mail: mccossu@uniss.it.

Il saggio costituisce l'aggiornamento e lo sviluppo di un lavoro di ricerca che si è concluso con la pubblicazione dell'articolo 'Recesso dell'investitore e determinazione del danno risarcibile nella gestione di portifogli di investimento', in Banca, borsaa, titoli di credito, 2006 , II, pp. 67-82. 


\section{Introduzione}

II contratto di "gestione di portafogli di investimento" può dirsi ormai accreditato come schema negoziale tipico. ${ }^{1}$ Le peculiarità legate sia all'oggetto dell'attività che, soprattutto e in via decisiva, alla causa contrattuale, inducono, infatti, a ripudiare i tralatizi riferimenti al mandato e/o alla gestione fiduciaria che hanno caratterizzato i primi anni del dibattito dottrinale e le prime pronunce giurisprudenziali. ${ }^{2}$

È noto, in proposito, che la gestione bancaria di portafogli di investimento in nulla si differenzia rispetto alla gestione posta in essere da un'impresa di investimento-s.i.m. All'interno di uno statuto speciale che regola un'attività certamente riservata, il legislatore ha istituito, infatti, una parità di trattamento tra queste due tipologie di operatori abilitati. ${ }^{3}$ D'altra parte, quelle stesse peculiarità di causa e oggetto dell'attività svolta alle quali si è fatto poc'anzi cenno servono a distinguere nettamente la gestione di portafogli non solo dal mandato e dalla gestione fiduciaria ma anche dal deposito titoli in amministrazione, ossia dal contratto bancario tipico di cui all'art. 1838 c.c. ${ }^{4}$

Torna utile a comprendere le peculiarità di questo considerare che realizza l'affidamento di un patrimonio ${ }^{5}$ in vista di una gestione dinamica e diversificata, ${ }^{6}$ là dove la diversificazione attiene al contenuto dell'oggetto del contratto stesso, e quindi alle singole utilità che lo compongono. Le più svariate operazioni di investimento e disinvestimento si compongono, dunque, entro la prospettiva assiologicamente unitaria di un'amministrazione profittevole, mentre il "patrimônio" conserva una costante unitarietà nonostante queste ininterrotte mutazioni di struttura e dimensioni (GUERRERA, 1988, p. 735; COSSU, 2002-A, p. 199 ss.). La giurisprudenza ha spesso fatto riferimento al mandato, ${ }^{7}$ ma invero al di là della indiscussa elasticità del

In questo senso, già nella disciplina previgente il Testo Unico della finanza contenuto nel d. Igs. 24 febbraio 1998, n. 58 (sul quale v. infra, nt. 9), Guerrera (1988, p. 698 ss.), il quale già nel quadro normativo imperniato sulla I. 23 marzo 1983, n. 77, istitutiva dei fondi comuni di investimento aperti, riteneva emerso un concetto assiologicamente autonomo di gestione patrimoniale; Carbonetti (1992, p. 106); Lener (1994, p. 3) della voce. Con riferimento alla disciplina attualmente vigente v. Cossu (2002-A, passim e spec. 25 ss.); Cossu (2002-B, 1 ss.) della voce; Cossu (2011, p. 769 ss.); nella stessa direzione Costi-Enriques (2004, p. 373). In senso parzialmente diverso Magno (2011, p. 927), testo e nt. 24, che ribadisce l'afferenza del contratto di specie al mandato, pur tuttavia osservando che tale schema negoziale non può adeguatamente spiegare le peculiarità della causa nella gestione di portafogli.

Per una casistica delle problematiche applicative emerse con riferimento alla natura del rapporto gestorio, specie in ipotesi di insolvenza del gestores.i.m., nel corso del primo decennio di esperienza giurisprudenziale a partire dalla I. 2 gennaio 1991, n. 1, v. Cossu (2003, II, p. 303 ), testo e nt. 2-3. Anche nella giurisprudenza di merito più recente compaiono tralatizi riferimenti al mandato, peraltro poco utili a interpretare le obbligazioni nascenti a carico del gestore dal contratto di gestione di portafogli: v. da ultimo Trib. Roma, 3 marzo 2017, n. 4393, in http://il caso.it. Ovviamente il "ritorno all'antico" é stato provocato anche dal fatto che nella più recente definizione sintetica di gestione di portafogli che si ritrova nell'elenco dei servizi di investimento di cui all'art. 1, comma 5-quinquies del testo unico della finanza (v. infra, nt. 9), é ricomparso il riferimento al mandato discrezionale che caratterizza l'incarico del gestore nella gestione di portafogli (che però non é un semplice mandato, come si vedrà sopra nel testo, e sta qui l'equivoco della giurisprudenza). Per la disciplina della gestione di portafogli v. infra, nt. 9.

Lo osservava con riferimento all'art. 2 d. Igs. 23 luglio 1996, n. 415, o decreto "Euro- sim" (poi abrogato dal testo unico della finanza) Cera (1997, I, p. 714). L'articolo in questione stabiliva infatti, al comma $1^{\circ}$, che "l'esercizio professionale nei confronti del pubblico dei servizi di investimento è riservato alle imprese di investimento e alle banche [...].". Quanto al t.u.f., l'art. 1, comma 1o, lett. $r$ ), inserisce nel novero dei soggetti abilitati allo svolgimento delle attività di investimento, oltre a s.i.m. (rectius: imprese di investimento nel nuovo lessico del legislatore) e banche (se) autorizzate, s.i.c.a.v., società di gestione del risparmio e intermediari finanziari iscritti nell'elenco speciale di cui all'art. 107 d. Igs. 10 settembre 1993, n. 385, o t.u.b. Soltanto le imprese di investimento e le banche, tuttavia, dispongono di una abilitazione generale allo svolgimento di tutti i servizi di investimento, mentre gli altri operatori sono abilitati solo rispetto ad alcune attività di intermediazione. Sul punto v. Cossu (2002-B, p. 76 ss.).

Dispone l'art. 1838, comma 10, c.c., che "la banca che assume il deposito di titoli in amministrazione deve custodire i titoli, esigerne gli interessi o i dividendi, verificare i sorteggi per l'attribuzione di premi o per il rimborso di capitale, curare le riscossioni per conto del depositante, e in generale provvedere alla tutela dei diritti inerenti ai titoli. Le somme riscosse devono essere accreditate al depositante". Sulla differenza tra la fattispecie in questione e la gestione di portafogli di investimento v. Cossu (2002-A, p. 148 ss.).

Si ha un'ipotesi di destinazione di (parte di) un patrimonio ad uno scopo: fra gli altri cfr. Bianca (1996, p. 154 ss.).

Il t.u.b., pervero, non opera una scelta concettuale definit(iv)a tra patrimonio autonomo e patrimonio separato: sul punto v. M. Cossu (nt. 1), 56 , testo e nt. 147. In argomento si rinvia ex pluris a Santoro Passarelli (1968, p. 86); Biondi (1956, p. 121 ss.). Per un'opinione che svaluta la distinzione tra l'una e l'altra categoria concettuale ritenendo che sia scarsamente significativa dal punto di vista dogmatico-ricostruttivo v. Pino (1950, p. 2).

V. Trib. Catania, 5 aprile 2004, in Banca, borsa, tit. credito (2006, II, p. 61), con nota di Cossu, Recesso dell'investitore e determinazione del danno risarcibile nella gestione di portafogli di investimento. II Tribunale rinviava, in particolare, all'art. 1710 c.c. per significare che "[...] in tema di mandato conferito al fine della gestione di titoli azionari, la diligenza dell'agente-mandatario deve assumere un connotato particolarmente rigoroso, e ciò in considerazione tanto della natura professionale e specializzata dell'incarico assunto che della posizione certamente dominante assunta dall'intermediario [...]". Nella fattispecie gli attori avevano stipulato con la Monte dei Paschi di Siena un contratto di gestione di portafogli, con annesso contratto di deposito titoli, dal quale recedevano due mesi dopo la stipula adducendo intervenute modifiche dell'assetto contrattuale e richiedendo l'accredito della corrispondente liquidità su un conto corrente acceso presso la medesima banca. Lamentavano, in proposito, ritardi nell'accredito richiesto, omissioni di rendiconto e un'ultrattività della gestione patrimoniale, che si sarebbe protratta oltre la data del recesso. La convenuta eccepiva di avere gestito il patrimonio con diligenza e nel rispetto della linea di investimento prescelta dagli investitori; eccepiva, ulteriormente, che il prematuro recesso e l'alea di gestione connaturata al contratto sarebbero stati, piuttosto, causa delle perdite sofferte dagli attori. Questi ultimi chiedevano invece che il Tribunale dichiarasse l'inadempimento della banca, applicando così gli artt. 1218 ss., c.c., e condannasse la stessa al risarcimento dei danni. In questa stessa ottica di aggancio normativo al mandato si spiega il riferimento operato, in materia di diligenza del gestore, a Cass. civ., 26 febbraio 1998, n. 2079 (si v. pubblicata in Giust. civ., 1998, I, p. 929 ss.), ossia ad una fattispecie pura di mandato conferito ad una banca per la compravendita di azioni. 
relativo schema negoziale è attestato che la gestione di portafogli non si avvantaggia granché dell'aggancio al mandato, se non quale schema di disciplina residuale, poiché la disciplina specifica dedicata al contratto di gestione di portafogli dall'art. 24 del testo unico della finanza e dai regolamenti della Consob é molto completa. ${ }^{9}$ Al di là, infatti, della comune attitudine a realizzare una forma di cooperazione gestoria la gestione di portafogli si distingue dal mandato per la sua natura di attività, intesa come quid pluris rispetto al mero compimento di (più) atti giuridici, ${ }^{10}$ fermo restando, poi, che l'imputazione del risultato al dominus costituisce, come nel mandato con rappresentanza, effetto legale automatico del contratto. ${ }^{11} \mathrm{E}$ irrilevante, invece, la natura dei beni che di volta in volta entrano a fare parte di questo "patrimônio", ${ }^{12}$ contando unicamente il loro valore, quale "astratta potenzialità patrimoniale". ${ }^{13}$ Alla luce della ricostruzione normativa, in particolare quella data dalle norme di rango primario, la gestione individuale di portafogli rappresenta dunque un contratto nominato e tipico ${ }^{14}$ che ha ad oggetto la prestazione di un servizio di investimento. ${ }^{15}$ A tale contratto si applica la disciplina speciale sui servizi di investimento contenuta nel testo unico della finanza e nelle norme di rango secondario emanate dall'autorità di vigilanza (Consob); solo in via residuale trova applicazione la disciplina di diritto comune in materia di mandato professionale: in particolare le regole di correttezza e diligenza nell'esecuzione dell'incarico ivi contenute (TOPINI, 1999, p. 713); infine, e incidentalmente si applica anche la disciplina sui contratti del consumatore di cui agli art. $33 \mathrm{ss}$. del codice del consumo contenuto nel decr. legisl. 6 settembre 2005, n. 206, ${ }^{16}$ come (peraltro) impone il diritto europeo (GIOIA, 2011, p. 68 ss.). Ma ciò accade solo se é presente il presupposto soggettivo nella controparte dell'impresa di investimento, cioé se si tratta di un consumatore.

Nei casi in cui il cliente di un contratto di gestione di portafogli citi in giudizio l'impresa di investimento lamentando che il contratto ha avuto un cattivo esito, spetta al cliente dimostrare l'inadempimento contrattuale. II giudice deve dunque in primo luogo verificare se ci sia stato l'inadempimento, e se ci sia un danno patrimoniale effettivo nella sua duplice componente di "perdite" (danno emergente) e di "mancato guadagno" (lucro cessante). Quest'ultima voce di danno viene riconosciuta, però, limitatamente alla misura

9 In argomento cfr. Luminoso (1985, p. 41 s.); Bavetta (1965, p. 322), in specie con riferimento alla duttilità dello schema negoziale. Evidenzia, invece, il logoramento dello schema dovuto (proprio) a questa sua dilatazione Alcaro (1988, p. 2 ss.) II Testo Unico della finanza italiano é contenuto nel d. Igs. 24 febbraio 1998, n. 58, modificato da ultimo con il d. legisl. 12 agosto 2016, n. 176. L'art. 24 del testo unico della finanza (anche t.u.f.) recita che "1. Al servizio di gestione di portafogli si applicano le seguenti regole: a) il cliente può impartire istruzioni vincolanti in ordine alle operazioni da compiere; b) i cliente può recedere in ogni momento dal contratto, fermo restando il diritto di recesso dell'impresa di investimento...ai sensi dell'articolo 1727 del codice civile; c) la rappresentanza per l'esercizio dei diritti di voto inerenti agli strumenti finanziari in gestione può essere conferita all'impresa di investimento...con procura da rilasciarsi per iscritto e per singola assemblea... 4 . Sono nulli i patti contrari alle disposizioni del presente articolo. La nullità può essere fatta valere solo dal cliente». Per la disciplina di dettaglio si v. l'art. 38 del regolamento intermediari (sul quale v. infra, nt. 38).

10 Cfr. Santagata (1985, p. 54), con particolare riferimento alla circostanza che l'art. 1703 cod. civ. ha ad oggetto il compimento di uno o più atti giuridici, non di un'attività. Sulla nozione di attività, e sulla relazione concettuale intercorrente tra atti e attività in diritto privato si rinvia a Alcaro (1995, p. 417 ss.); cfr. Oppo (1991, I, p. 475 ss.), ora in ID. (2000, p. 17), ove l'A. osserva la diversità dei principi che governano le attività rispetto agli atti, "[...] in ragione non solo del collegamento e coordinamento in esse di una molteplicità e, spesso, varietà di atti ma della combinazione di momenti giuridici e di parimenti essenziali momenti tecnici, del loro protrarsi nel tempo, del maggior coinvolgimento di interessi generali o di terzi". Quanto alle zone d'ombra che tuttora insistono sulla nozione di attività, ma anche alla sua utilità sul piano empirico, v. Rondinone (2001, p. 3 ss.)

1 Cossu (2002-A); Guerrera (1988, p. 764 ss.), il quale già osservava che detta imputazione si verifica "[...] in virtù di una fattispecie complessa a formazione progressiva, che ingloba, quali concause necessarie e cronologicamente distinte dell'effetto ultimo, sia il contratto di investimento (che funge da quadro programmatico), sia il singolo atto gestorio (che ne rappresenta un momento attuativo)".

12 È evidente che il termine "patrimonio" viene usato in senso lato, posto che è irrilevante allo schema negoziale in esame l'appartenenza o meno, in senso giuridico, all'investitore del portafogli affidato in gestione, ossia la circostanza che l'investitore ne sia stricto sensu proprietario.

13 Guerrera (1988, p. 734 ss.); v. Anche Lener (1994), ove l'osservazione che interessa all'investitore qualunque bene monetizzabile assunto come valore, non già uno specifico bene in luogo di altri.

14 Cossu (2002-A, p. 227 ss.). Si può, poi, incidentalmente ricordare che la tipicità, pur in assenza di esplicite prescrizioni normative in questo senso, può essere conferita (solo) da norme di rango primario, come si desume dall'art. 1322, comma 1o, c.c.

15 Sulla natura di "servizio" e non di "strumento" di investimento della gestione di portafogli v. le convincenti considerazioni di Bochicchio (1997, I, p. 1075). La gestione, infatti, "[...] concretizzandosi in ripetuti investimenti e disinvestimenti, al fine di valorizzare un determinato patrimonio, [...] ha a propria volta per oggetto i singoli strumenti". Nello stesso senso, già con riferimento alla gestione di patrimoni mobiliari di cui all'art. 8 I. 2 gennaio 1991, n. 1, Lener (1996, p. 79), negando la possibilità di considerare l'operazione di investimento come valore mobiliare autônomo. (FAZIO, 2011, p. 82).

16 In argomento, e in questa prospettiva dell'applicabilità all'intero settore dei servizi di investimento della disciplina sulle clausole vessatorie di cui agli artt. 1469 bis e seguenti cod. civ., (poi abrogati e sostituiti dagli artt. 33 s.s. del codice del consumo) v. Gabriellia e Orestano (2000, p. 225 ss.); Rovito-Picardi (2002, p. 202). Può affermarsi, in questo senso, la natura transtipica della disciplina relativa ai contratti del consumatore: Roppo (2001, p. 770 ss.). In ogni caso è deprecabile il silenzio del t.u.f. in ordine al raccordo tra la disciplina dei servizi di investimento e quella sulle clausole abusive: v. Alpa (1998, t. I, p. 261 ss.). In giurisprudenza, sull'applicabilità della disciplina sulle clausole vessatorie di cui agli artt. 1469-bis-1469 sexies c.c., e dello "statuto dei diritti del consumatore" di cui alla I. 30 luglio 1998, n. 281, ai contratti di negoziazione, collocamento e raccolta di ordini concernenti valori mobiliari (rectius: leggi "strumenti finanziari"), v. App. Roma, 24 settembre 2002 , in Corr. giur., 2003, p. 471 ss., con nota di Fici (2004, I, p. 1618 ss.). 
del guadagno virtuale che gli investitori avrebbero goduto in caso di tempestiva liquidazione del portafogli, mentre si nega il risarcimento "[...] di un pregiudizio sofferto diverso ed ulteriore rispetto a quello verificatosi al momento del recesso dal contrato", perché il recesso é l'evento che segna la fine del rapporto e quindi rappresenta anche "[...] la data sino alla quale calcolare la sussistenza di un eventuale lucro cessante imputabile all'inadempimento contrattuale della banca [...]" (e non oltre).

\section{Diligenza professionale e violazione delle istruzioni vincolanti impartite dall'investitore}

Non si può qui ripercorrere l'imponente dibattito presente alla dottrina in materia di diligenza professionale del gestore, coerente alla natura altamente professionale dell'incarico, ${ }^{17} \mathrm{e}$ in materia di regole tecniche e comportamentali da osservare nell'esercizio dell'attività. ${ }^{18}$

Nell'esame di merito del singolo caso concreto da parte del Tribunale (quindi nel primo grado del giudizio) é fondamentale verificare se l'investitore aveva impartito istruzioni vincolanti, che delimitavano la discrezionalità e l'autonomia operativa del gestore nella selezione delle operazioni di investimento e disinvestimento.

Ė appena il caso di rammentare che l'obbligazione assunta dall'impresa di investimento viene pressoché concordemente classificata dalla dottrina come obbligazione di mezzi e non di risultato. ${ }^{19}$ Sotto il profilo della valutazione dell'inadempimento del rapporto obbligatorio, tale è, certamente, "[...] l'andare oltre i termini della gestione e gli obiettivi della stessa [...]"; ciò che rappresenta ad ogni effetto un "difetto nell'esecuzione del contrato" (MASTROPAOLO, 2003, p. 577). L'alto grado di competenze tecniche richieste al gestore per l'espletamento dell'attività non prelude, dunque, ad un'obbligazione di risultato, bensì semplicemente segnala che l'attività dedotta in oggetto sociale è speciale, oltre che, può aggiungersi, riservata. ${ }^{20}$ L'art. 24, comma 10, lett. $b$ ), del testo unico della finanza stabilisce espressamente il diritto dell'investitore a impartire istruzioni vincolanti, con l'effetto di circoscrivere il contenuto dell'oggetto del contratto e con la conseguenza che la violazione delle istruzioni rappresenta un vero e proprio inadempimento contrattuale..$^{21}$

La presenza di un precetto di rango speciale, qual è appunto l'art. 24 del testo unico della finanza, rende superfluo, poi, il riferimento all'art. $1197 \mathrm{cod}$. civ., e dunque all'ipotesi di adempimento dell'obbligazione

17 Si v. Cossu (2001, p. 589 ss.); nel campo dei servizi di investimento la diligenza è certamente inclusiva di una certa dose di perizia: si v. Lobuono (1999, p. 179 ss.)

18 I riferimenti vanno, principalmente, quanto al t.u.f. all'art. 21, sui criteri generali di condotta; all'art. 22, sulla separazione patrimoniale; all'art. 23, sulle regole per la redazione dei contratti; all'art. 24, dedicato alla gestione di portafogli; quanto al regolamento intermediari (sul quale v. meglio infra, nt. 32), all'art. 26, sulle regole generali di comportamento del gestore; all'art. 28, sullo scambio di informazioni tra gestore e investitore; all'art. 30 , sui contratti con gli investitori.

19 La distinzione tra obbligazioni di mezzi e obbligazioni di risultato, o per meglio dire la sua rilevanza ricostruttiva, per la verità non è pacifica in dottrina: in argomento si rinvia a Mengoni (1954, I, p. 185 ss.), dove l'A. ripercorre le tappe che hanno condotto la dottrina francese ad elaborare le due categorie contestandone non tanto la fondatezza in astratto quanto l'utilità pratica, data la difficoltà di precisare i concetti stessi di "mezzo" e "risultato". In ogni caso, prevale l'opinione che quella del gestore professionale sia un'obbligazione di mezzi: v. ex pluris Morozzo Della Rocca, $(1991$, p. 870 ss.). Si evidenzia, tuttavia, l'impossibilità di tracciare una linea distintiva nettissima tra le une e le altre, posto che la ricorrenza di regole tecniche a contenuto altamente individuante della condotta richiesta talvolta avvicina la prestazione di mezzi all'area delle obbligazioni di risultato: si v. recentemente Salvatore $(2004$, p. 338). In giurisprudenza, ha ritenuto che la gestione di un portafogli titoli su base discrezionale faccia sorgere in capo alla banca un'obbligazione di mezzi Trib. Roma, 9 dicembre 1991, in Giust. civ., (1992, I, p. 1355 ss.), con riferimento ad una controversia insorta prima dell'entrata in vigore della I. 2 gennaio 1991, n. 1; nello stesso senso cfr. App. Paris, 19 mai 1998 , n. 184 , in Riv. trim. droit comm., 1998, 893 ss., con riferimento alla fattispecie, omologa a quella descritta nell'art. 24 t.u.f. italiano, di cui all'art. 64 , alinea 1o, L. 2 juillet 1996.

20 Essombè Moussio (1996, § 350): « [...] la spécialisation et l'autonomie du métier de la gestion pour compte tiers paraissent un processus inéluctable $[\ldots] »$.

21 In questo senso, tra gli altri, Picardi (2004, p. 467); Mastropaolo (2003); cfr. Magno (2011, p. 934 s.). In generale sul rapporto tra art. 1218 e art. 1176 c.c. v. ex pluris Visintini-Cabella Pisu (1986, p. 166). In giurisprudenza, con riferimento al carattere vincolante delle istruzioni impartite dall'investitore cfr. Trib. Biella, 24 gennaio 2001, in Giur. it. (2002, I, p. 2332), con nota di Gaeta (2002, II, p. 242 ss.), con nota di Maccabruni, Evoluzione normativa dei doveri della banca nei contratti di gestione patrimoniale attraverso operazioni su strumenti finanziari. La pronuncia in questione, peraltro, è richiamata da Trib. Catania, 5 aprile 2004, (cit., v. nt. 8), a sostegno dell'applicabilità delle norme sul mandato alla banca nell'esecuzione di operazioni in conto corrente, ex art. 1856 c.c. Nello stesso senso anche Trib. Mantova, 12 novembre 2004 , in Contr. (2005, p. 583 ss.), con nota di GAETA, Responsabilità oggettiva degli intermediari e validità dei contratti di investimento. 
a mezzo di prestazione qualitativamente inesatta, ${ }^{22}$ così come il richiamo all'art. 1711 cod. civ., e quindi all'obbligo del mandatario di rispettare i limiti imposti per contratto alla propria azione,$^{23}$ ovvero ancora alla figura del mandato speciale elaborata dalla giurisprudenza. ${ }^{24}$

Il problema principale, in quest'ipotesi, è stabilire in quale misura l'inosservanza delle istruzioni si sia rivelata dannosa per il portafogli in gestione; ciò che richiederebbe, come accade in altri ordinamenti, una valutazione prognostica (e) comparativa tra i risultati gestori ottenuti e quelli cui ragionevolmente si sarebbe pervenuti là dove il gestore avesse rispettato le istruzioni dell'investitore ${ }^{25}$ valutazione che rende sostanzialmente imprescindibile un apprezzamento del caso concreto attraverso la contemplazione delle variabili che personalizzano la prestazione dovuta precisandone i contenuti con riferimento alla persona del singolo investitore. ${ }^{26} \mathrm{Nel}$ complesso, dunque, lo standard di diligenza si determina non solo in ragione di regole di condotta oggettive, individuate dalle norme di legge e regolamentari, ma anche in ragione di regole con valenza ed efficacia individuali che discendono dalle (eventuali) istruzioni che integrano il contenuto dell'oggetto del contratto; queste ultime personalizzano la prestazione professionale in ragione delle caratteristiche e delle aspettative del suo destinatário (TOPINI, 1999, p. 714).

Bisogna dire che nella materia de qua manca ancora l'elaborazione di un completo diritto giurisprudenziale, riscontrandosi orientamenti diversificati nella giurisprudenza. ${ }^{27}$

Un elemento che incide profondamente sul giudizio di diligenza é se gli investitori - attori siano "clienti al dettaglio" (o clienti retail) oppure "investitori esperti (e precisamente "clienti professionali" o "controparti qualificate")', posto che solo per i primi il regolamento Consob sugli intermediari ha dettato una protettiva: in particolare, non risulta che avessero documentato, e quindi in qualche modo "autocertificato", la propria competenza in materia di servizi e strumenti finanziari. ${ }^{28}$ Tra l'altro, ai sensi dell'art. 23 , comma 1o, del testo

22 Dispone l'art. 1197, comma 10, c.c., che "il debitore non può liberarsi eseguendo una prestazione diversa da quella dovuta, anche se di valore uguale o maggiore, salvo che il creditore consenta. In questo caso l'obbligazione si estingue quando la diversa prestazione è eseguita". Si rinvia in argomento a Breccia (1991, p. 407 ss.).

23 Dispone l'art. 1711, comma 1o, c.c., che "il mandatario non può eccedere i limiti fissati nel mandato. L'atto che esorbita dal mandato resta a carico del mandatario, se il mandante non lo ratifica".

24 Prima dell'entrata in vigore della I. n. 1/91 a proposito del mandato conferito per la gestione di titoli azionari parlava di mandato speciale con riferimento all'ipotesi in cui il mandante avesse fornito istruzioni specifiche circa l'esecuzione dell'incarico, tra le altre, Cass., 15 gennaio 2000 , n. 426 , in Contr., 2000, p. 911 ss., con nota di ANGIOLI, Responsabilità del mandatario per la gestione azionaria.

25 Lobuono (2002, p. 269), anche per ampi richiami alla dottrina tedesca, che in casi simili ritiene che l'inadempimento del gestore assuma rilevanza causale "[...] limitatamente al danno corrispondente alla differenza fra il pregiudizio provocato all'investitore e quello che si sarebbe comunque verificato ove fossero state rispettate le guidelines [...]".

26 Cfr. in questo senso Com., 24 février 1998 e ID., 7 avril 1998, in Riv. trim. droit comm. (1998, p. 637 ss.), con riferimento segnatamente alle variabili rappresentate dalla situazione finanziaria dell'investitore, dai suoi obiettivi di investimento, dalla sua esperienza e competenza in materia di servizi e strumenti finanziari, dal suo essere o meno avvertito dei rischi connessi ad operazioni speculative.

27 Si v. Magno (2011, p. 951, testo e nt. 92). Nella giurisprudenza di merito, v., di recente, Trib. Roma, 25 maggio 2005 , in Corr. Giur. (2005, p. 1275 ss.), con nota di Di Majo, Prodotti finanziari e tutela del risparmiatore. Osserva il Tribunale che l'intermediario autorizzato - nella fattispecie una banca - «[...] non è mero e passivo esecutore degli ordini di acquisto del cliente ma ha il preciso obbligo di informarlo delle caratteristiche specifiche dell'operazione (comunicandogli e spiegandogli, tra l'altro, il grado di affidabilità del titolo, secondo le agenzie di rating) e della eventuale non-adeguatezza dell'investimento richiesto [...] ».

28 II regolamento sulla disciplina degli intermediari è contenuto nella delibera Consob 29 ottobre 2007, n. 16190, ed é stato poi modificato varie volte, e da ultimo con con delibera Consob 17 marzo 2016, n. 19148. L'Allegato III al regolamento elenca i "clienti professionali privati", e distingue i "clienti professionali privati di diritto" (ovvero classificati come clienti professionali per qualunque servizio e strumento di investimento): "(1) i soggetti che sono tenuti ad essere autorizzati o regolamentati per operare nei mercati finanziari, siano essi italiani o esteri quali: a) le banche; $b$ ) le imprese di investimento; $c$ ) altri istituti finanziari autorizzati o regolamentati; $d$ ) le imprese di assicurazione; e) gli organismi di investimento collettivo e le società di gestione di tali organismi; $f$ ) i fondi pensione e le società di gestione di tali fondi; $g$ ) i negoziatori per conto proprio di merci e strumenti derivati su merci; $h$ ) i soggetti che svolgono esclusivamente la negoziazione per conto proprio su mercati di strumenti finanziari e che aderiscono indirettamente al servizio di liquidazione, nonché al sistema di compensazione e garanzia (locals); $i$ ) altri investitori istituzionali; $I$ ) gli agenti di cambio; (2) le imprese di grandi dimensioni che presentano a livello di singola società, almeno due dei seguenti requisiti dimensionali: - totale di bilancio: 20000000 EUR, - fatturato netto: 40000 000 EUR, - fondi propri: 2000000 EUR". L'Allegato III classifica anche i "clienti professionali privati su richiesta". Può ottenere tale qualificazione e conseguente classificazione qualunque soggetto che, oltre ad espletare la procedura di richiesta secondo le modalità indicate nell'Allegato, attesti il possesso di almeno due tra i seguenti requisiti: avere effettuato operazioni di dimensioni significative sul mercato con una frequenza media di 10 operazioni al trimestre nei quattro trimestri precedenti; — detenere un valore di portafoglio in strumenti finanziari, inclusi i depositi in contante, che supera i 500.000 EUR; - lavorare o avere lavorato nel settore finanziario per almeno un anno in una posizione professionale che presupponga la conoscenza delle operazioni o dei servizi previsti. La classificazione dei clienti professionali pubblici é contenuta invece in un regolamento ministeriale. L'art. 2, comma 1, d.m. Ministero economia e finanze (MEF) 11 novembre 2011, n. 236, elenca i "clienti professionali pubblici" ai sensi dell'art. 6, comma 2-sexies, testo unico della finanza (sul quale v. supra, nt. 9), e subclassifica i "clienti professionali pubblici di diritto", ovvero classificati come clienti professionali pubblici per tutti i servizi anche di gestione collettiva e per tutti gli strumenti finanziari, il Governo della Repubblica e la Banca d'Italia. Sono, invece, « clienti professionali pubblici su richiesta », ai sensi dell'art. 3 del decreto, le Regioni, le Province autonome di Trento e Bolzano, i soggetti di cui all'art. 2 d. Igs. 18 agosto 2000, n. 267 (enti locali), nonché gli enti pubblici nazionali e regionali, a condizione che i richiedenti soddisfino congiuntamente i seguenti requisiti: a) entrate finali accertate nell'ultimo rendiconto di gestione approvato superiori a 40 milioni di euro; $b$ ) operazioni effettuate sul mercato finanziario di valore nominale o nozionale complessivo superiore a 100 milioni di euro nel corso del triennio precedente la stipula del contratto; c) presenza in organico di personale addetto alla gestione finanziaria che abbia acquisito adeguate competenze, conoscenze ed esperienza in materia di servizi di investimento, ivi compresi quelli di gestione collettiva, e strumenti finanziari". 
unico della finanza, in ragione della natura professionale dei contraenti può essere derogato l'obbligo di redazione del contratto in forma scritta. ${ }^{29}$

Certamente né la disciplina di rango primario né la disciplina regolamentare consentono di limitare la possibilità per l'investitore di agire in via risarcitoria a seconda dell'esperienza e/o delle conoscenze che vanti in servizi e strumenti finanziari. ${ }^{30}$

Certamente se l'operatore ha a che fare con un investitore esperto ciò incide sull'onere probatorio, nel senso che l'osservanza delle regole di condotta, e segnatamente della diligenza professionale, potrebbe essere valutata con minore rigore. ${ }^{31}$

Fattori ulteriori che possono incidere sulle modalità espressive del giudizio di diligenza sono le "caratteristiche della gestione", che deve rispondere al parametro della sua adeguatezza, cioè deve tenere conto sia delle caratteristiche finanziarie del cliente che delle sue condizioni patrimoniali ${ }^{32}$ e della sua propensione al rischio.

E' evidente che l'adeguatezza é una clausola generale-parametro, in quanto stima il grado di diligenza professionale del gestore, e nel contempo segna anche il perimetro della sua responsabilità contrattuale. E' un parametro di livello particolarmente elevato che si utilizza solo nei contratti a più elevato valore aggiunto, che sono la gestione di portafogli e la consulenza in materia di investimenti, mentre $\mathrm{i}$ contratti di negoziazione e mediazione sono soggetti alla clausola generale-parametro di appropriatezza, che considera la specifica conoscenza ed esperienza del cliente in materia di strumenti finanziari e servizi di investimento, non anche un giudizio di coerenza con il profilo di rischio del cliente e con la sua situazione finanziaria. Quanto poi ai servizi "minori”, quali sono la ricezione e trasmissione di ordini e l'esecuzione di ordini per conto della clientela, dato il basso livello di valore aggiunto che l'attività del gestore fornisce a questi servizi elementari, essi sono eseguiti in modalità c.d. execution only.

I tre parametri, in ordine descrescente di raffinatezza - dell'”adeguatezza", dell'appropriatezza, della execution only - rappresentano le tre modalità alternative espressive del canone generale di best execution e dei suoi corollari della know your customer rule e della suitability rule (nell'interesse dei clienti e del mercato) sancito dalla MiFID e confermato nella normativa di secondo livello così come ora dalla MiFID II, che entrerà in vigore nei paesi dell'Unione Europea il 3 gennaio $2018 .{ }^{33}$

II gestore deve quindi selezionare le tipologie di strumenti finanziari che può utilizzare dentro la gestione del portafogli in ragione dei tre elementi di cui sopra, attenersi alle eventuali istruzioni vincolanti impartite dal cliente e scegliere obbligatoriamente (se il cliente é retail) un benchmark al quale ancorare ex ante l'andamento della gestione (e al quale parametrare ex post il suo rendimento). ${ }^{34}$

29 A meno che il cliente professionale non abbia chiesto di essere trattato come un cliente al dettaglio, o retail, perchè in questo caso l'obbligo di redigere il contratto in forma scritta rimane.

30 II Regolamento intermediari ha optato per un sistema «a gradini», o «graduata» (ANNUNZIATA, 2015, p. 150; AMOROSINO, 2014, p. 99), in virtù del quale lo stesso cliente può dichiararsi esperto per talune operazioni e rispetto ad alcuni prodotti o strumenti finanziari, non necessariamente per tutti: si v., all'interno del regolamento intermediari contenuto nella delibera Consob 29 ottobre 2007, n. 16190, l'art. 35, in materia di “informazioni riguardanti la classificazione dei clienti". Ciò significa che il cliente professionale può chiedere di essere classificato come cliente retail per uno o più prodotti, strumenti o servizi.

31 Taluni auspicano, in particolare, una maggiore sensibilità del legislatore alla distinzione tra investitori sofisticati e investitori comuni, anche sul piano dell'elaborazione di diversi modelli di responsabilità: (LOBUONO, 2002, p. 223, testo e nt. 56; GIOIA, 2011, p. 68).

32 Il parametro dell'adeguatezza é sancito dall'art. 40 del regolamento intermediari (delib. Consob n. 16190/2007, cit. supra, in nt. 32). Siv. sul tema, tra gli altri. (SARTORI, 2008, p. 25 ss.). Alla propensione al rischio dell'investitore fa esplicito riferimento l'art. 40, comma 1o, lett. b) del regolamento intermediari, in materia di "adeguatezza" (e v. supra, nt. 36).

33 Art. 21 direttiva 21 aprile 2004, n. 2004/39/CE (MiFID); artt. 44, 45, 46 direttiva 2006/73/CE; art. 27 direttiva 15 maggio 2014, n. 2014/65/UE (MiFID II). La Direttiva MiFID II enterà in vigore il 3 gennaio 2018, insieme al Regolamento (UE) n. 600/2014 del Parlamento europeo e del Consiglio, del 15 maggio 2014, sui mercati degli strumenti finanziari, che modifica il regolamento (UE) n. 648/2012 (MiFIR) in merito tra l'altro ai mercati su derivati. Si osserva, tuttavia, come già nel vigore della disciplina antecedente la normativa comunitaria incentrata sulla direttiva ISD e il testo unico della finanza (v. supra, nt. 1), ovvero nella prima legge s.i.m. (I. 2 gennaio 1991, n. 1), l'art. 24, comma 1, del regolamento intermediari (contenuto nella delibera Consob 2 luglio 1991, n. 5387) sostanzialmente codificava il principio di best execution: (LUCANTONI, 2011, p. 270, nt. 58).

34 L'art. 38, comma 1, lett. a), del regolamento intermediari prescrive che il contratto di gestione, se la controparte é un cliente retail, deve precisare quali tipologie di strumenti finanziari possono essere incluse nel portafogli, e i tipi di operazioni che possono essere compiute su questi strumenti finanziari, e i limiti (ad esempio se l'intermediario può utilizzare l'effetto leva). E si veda poi l'art. 29, comma 2, del regolamento, che stabilisce che qualora la controparte sia un cliente retail l'intermediario deve utilizzare nella gestione di portafogli un parametro oggettivo di riferimento - o benchmark - coerente con i rischi connessi alla gestione stessa, e deve commisurare i risultati della gestione al parametro, in modo da consentire al cliente di valutare la gestione. 
Sotto il profilo gestorio è evidente, peraltro, che la presenza di un parametro scelto contrattualmente per la stima dell'andamento della gestione condiziona la politica di asset allocation anche per quanto riguarda la selezione degli strumenti finanziari, la possibilità di ricorso a prodotti e strumenti derivati, illiquidi o altamente volatili.

Il contratto definisce, infine, gli obblighi informativi periodici da fornire alla clientela.

\section{Categorie di inadempimento}

Le tipologie di inadempimento che più spesso provocano un contenzioso e danno occasione a pronunce della giurisprudenza sono a) l'ultrattività della gestione, cioè il caso particolare in cui il gestore ha continuato a gestire il portafogli anche dopo il recesso del cliente dal contratto (recesso che secondo l'art. 24 , comma 10, lett. $d$ ), del testo unico della finanza è possibile in qualunque momento e senza penalità o spese) e per effetto di questo indebito protrarsi dell'esecuzione del contratto le perdite del portafogli si sono aggravate; $b$ ) l'omissione dellinformazione periodica al cliente sulle operazioni eseguite; $c$ ) l'abuso gestorio, ovvero il caso in cui il gestore (la banca o l'impresa di investimento) ha violato le istruzioni fornite dal cliente-investitore.

In tutti i casi é normale che il Tribunale abbia necessità di avvalersi di una consulenza tecnica di ufficio. ${ }^{35}$

Nel primo caso (quello indicato sub a)) la prosecuzione dell'attività di gestione da parte della banca o dell'impresa di investimento dopo il recesso del cliente rappresenta dal punto di vista negoziale una contravvenzione al divieto di intraprendere nuove operazioni. Se il gestore in questo caso prosegue normalmente l'attività si realizza, come si é appunto detto, una situazione di ultrattività della gestione che in assenza di regolamentazione specifica ${ }^{36}$ può essere ricondotta per analogia juris all'art. 1398 cod. civ. o all'art. 1711 cod. civ., e dunque intesa come eccesso di potere di rappresentanza o come eccesso di potere gestorio ${ }^{37}$ - oppure anche congiuntamente entrambi, posto che il gestore nell'esercizio dell'attività oltre che un potere di gestione esplica anche un potere di rappresentanza. In applicazione dei principi di diritto comune l'eccesso di potere di rappresentanza dà luogo pertanto a responsabilità, a meno che non intervenga una ratifica dell'interessato secondo l'art. 1399 cod. civ.

In alternativa potrebbe forse parlarsi ai sensi dell'art. 2028 cod. civ. di una gestione di affari altrui intesa in senso ampio, secondo l'interpretazione lata dell'art. $2028 \mathrm{cod}$. civ. che è stata proposta da alcuni Autori; ${ }^{38}$ ma in realtà, nonostante l'attuale e ben nota tendenza a ridimensionare e svalutare la distinzione tra responsabilità contrattuale e responsabilità aquiliana, resta il fatto che in virtù del rapporto contrattuale (già) intercorso tra gestore e investitore anche le operazioni compiute dopo il manifestarsi dell'efficacia

35 In particolare per accertare se il gestore ha violato la linea di gestione prescelta dal cliente, se ha destinato la provvista, in tutto o in parte, ad investimenti su strumenti finanziari non contemplati dal contratto, oppure esorbitanti rispetto al profilo di rischio del cliente. Sui limiti oggettivi che la consulenza tecnica di ufficio presenta in materia di contratti di intermediazione finanziaria v. infra, nt. 73.

36 Secondo l'art. 24, comma 1, lett. b), del testo unico della finanza (sul quale v. supra, nt. 9) "il cliente può recedere in ogni momento dal contratto, fermo restando il diritto di recesso dell'impresa di investimento, della società di gestione del risparmio o della banca a sensi dell'articolo 1727 del codice civile".

37 In questo senso, con riferimento al compimento, da parte del gestore, di operazioni che impegnino l'investitore oltre i limiti del portafogli (SALANITRO, 1998, p. 186).

38 L'elemento caratteristico della fattispecie descritta nell'art. 2028 cod. civ. é che il gestore interviene senza esservi obbligato o richiesto; "[...] nondimeno, la norma è stata interpretata dalla giurisprudenza con una certa larghezza. A ben vedere, difatti, l'ingerenza può ritenersi non autorizzata (e quindi "spontanea"), anche quando avvenga in occasione dell'esecuzione di uno specifico rapporto obbligatorio, ma ne ecceda i limiti convenzionali e legali": (BRECCIA, 1982, p. 709. Sul tema v. già (ARU, 1957, p. 212), osservando che "[...] non sembra possa escludersi la contemporanea esistenza, nella stessa persona, della veste di gestore e di mandatario, naturalmente non rispetto allo stesso affare, ma ad affari diversi. II mandatario che tratta negozi eccedendo i limiti del mandato, e non in dipendenza di questo, può, quando abbia l'intenzione di gerire un affare altrui, essere considerato come gestore, né pare osti a questa conclusione il disposto dell'art. 1711 cod. civ., il quale implica che l'azione che eccede i limiti del mandato sia in relazione con questo". Sul tema, e per i confini della fattispecie della Geschäftsführung, si rinvia a Larenz (1972, spec., p. 270ss.). 
del recesso sono riconducibili all'alveo della responsabilità contrattuale ${ }^{39}$ e non della responsabilità per gestione d'affari altrui. Vale, infatti, ad allontanarle dall'area dell'illecito aquiliano la circostanza che si tratta pur sempre di operazioni (dalle quali è derivata una perdita) le quali sono direttamente collegabili agli effetti del contratto. ${ }^{40}$

\section{Quantificazione del danno emergente e del lucro cessante}

Ora, l'esame del giudice di merito deve accertare la sussistenza del danno emergente, cioé quantificare le perdite (e se per caso si é verificata una ultrattività della gestione dopo l'eventuale recesso del cliente, il giudice dovrà anche accertare la differenza tra il danno complessivo e il danno che si sarebbe verificato in ipotesi di tempestiva liquidazione del portafogli); e deve inoltre quantificare il lucro cessante o mancato guadagno. ${ }^{41}$ Riguardo invece al secondo tipo di violazioni (quello indicato nel paragrafo precedente sub $b$ )), bisogna dire che in materia di diritto di informazione il contratto non può precisare con assoluta esattezza le caratteristiche della relazione tra le parti. Questa obbligazione, infatti, sicuramente ha un contenuto fortemente tecnico ${ }^{42}$ ed esige regole giuridiche di condotta, però implica anche obblighi a contenuto variabile, quali sono quelli richiamati nelle clausole generali di buona fede (oggettiva) e di correttezza, ${ }^{43}$ come specificamente declinati nei contratti di intermediazione finanziari. ${ }^{44} \mathrm{Non}$ mancano, tuttavia, nella disciplina regolamentare norme che puntualizzano esattamente la frequenza del flusso informativo tra il gestore e il cliente ${ }^{45}$ ed è certo che il gestore é obbligato ai rendiconti periodici non solo dal punto di vista della relazione contrattuale con il cliente ma anche sotto il profilo della regolare tenuta delle scritture contabili, perché il rendiconto relativo al servizio di gestione é una scrittura contabile obbligatoria ${ }^{46}$ descritta nell'Allegato 5 al regolamento emittenti. ${ }^{47}$ L'obbligo riguarda dunque sia la redazione del rendiconto che l'invio al cliente nei tempi e con le modalità stabilite nel contratto; in caso contrario si realizza non solo una condotta negligente ma anche un'infrazione alla disciplina sulle scritture contabili obbligatorie.

Riguardo alla prova del danno, secondo un'opinione sufficientemente consolidata in dottrina l'inversione dell'onere probatorio rappresentata nell'art. 23, comma $6^{\circ}$, del testo unico della finanza, ${ }^{48}$ opera in un duplice senso, poiché da un lato l'investitore è esonerato dalla prova del nesso causale tra danno e

39 Sull'incerto solco tra responsabilità contrattuale e illecito aquiliano si rinvia a Maggiolo (2003, p. 90 ss.). E si può incidentalmente osservare che parte della dottrina ritiene che l'inversione dell'onere probatorio di cui all'art. 23, comma 6o, del testo unico della finanza (sul quale v. supra, nt. 9) sia applicabile anche alle azioni risarcitorie extracontrattuali: in questo senso, in particolare, Topini (1999, loc. ult. cit.)

40 Sul tema si rinvia a Maggiolo (2003, p. 271 ss.).

41 Per la distinzione tra danno emergente e lucro cessante si rinvia a De Cupis (1946, p. 150), il quale precisa che la distinzione sta nell'attualità o meno dell'interesse: "[...] se oggetto del danno è un interesse attuale, ovverosia l'interesse relativo a un bene già spettante ad una persona nel tempo in cui il danno medesimo è cagionato, si ha un danno emergente; se, viceversa, oggetto del danno è un interesse futuro, ovverosia l'interesse relativo a un bene non ancora spettante ad una persona, si ha un danno lucro cessante".

42 In materia di gestione di portafogli l'obbligazione del gestore non si esaurisce nel dare informazioni al cliente, poiché in cliente, soprattutto se non é un cliente professionale, necessita, ulteriormente, di essere consigliato dal gestore sul da farsi: "[...] da questa constatazione è sorta la convinzione che sull'intermediario gravi una più intensa obbligazione: il c.d. dovere di consiglio...È infatti tipico del rapporto tra un profano ed un iniziato che il primo attenda dal secondo un consiglio illuminante". (MOROZZO DELLA ROCCA, 1991, p. 889).

43 Ex pluris si veda Clement (1997, p. 203 ss.); Rodotà (1987, p. 715 ss.). In giurisprudenza, sulla difficoltà di precisare il contenuto del dovere di informativa nei contratti di gestione cfr. Trib. Mantova, 18 marzo 2004, in Banca, borsa, tit. credito, 2004 , II, p. 440 ss., con nota di Maffeis, Conflitto di interessi nella prestazione di servizi di investimento: la prima sentenza sulla vendita a risparmiatori di obbligazioni argentine.

44 Per il contenuto che le clausole generali assumono nel contesto specialistico dei mercati finanziari v. Venuti (2000, p. 1057 ss.), ove l'A. riguardo alla correttezza osserva che nella materia dei servizi finanziari il concetto di correttezza richiama e comprende sia il comportamento descritto negli artt. 1175 e 1375 del codice civile italiano, e sia anche il concetto anglosassone di fairness, che include l'imparzialità nell'etica degli affari. Quest'ultimo a sua volta evoca gli standard di efficienza e le regole di deontologia professionale, "[...] e cioè un agire rispettoso degli interessi dei concorrenti oltre che della generalità dei consumatori e utente".

45 Secondo l'art. 54, comma 3o, del regolamento intermediari contenuto nella delib. Consob n. 16190/2007 (supra, in nt. 32) nel contratto di gestione di portafogli destinato a clienti retail il rendiconto deve essere semestrale, a meno che il cliente non richieda un rendiconto trimestrale; e in ogni caso, cioè anche quando il cliente non richieda il rendiconto semestrale, deve essere inviato almeno ogni dodici mesi. Se il contratto di gestione prevede il ricorso all'effetto leva il rendiconto deve essere mensile.

46 II rendiconto è qualificabile come scrittura contabile obbligatoria ai sensi dell'art. 2214, comma 2o, cod. civ. (COSSU, 2002-A, p. 173 ss.).

47 Ad esempio per i gestori collettivi (società di gestione del risparmio-SGR e Sicav) si v. l'art. 2 del decreto del Ministero economia e Finanze (MEF) 5 marzo 2015, n. 30, che attua l'art. 39 del testo unico della finanza (sul quale v. supra, nt. 9) in materia di criteri generali ai quali devono uniformarsi gli organismi collettivi di investimento del risparmio (OICR) italiani (supra, in nt. 32) che in aggiunta alle scritture contabili richieste a tutte le imprese commerciali dal codice civile (art. 2214 ss.) i gestori devono redigere il libro giornale, una relazione annuale e una relazione semestrale sulle attività svolte e un prospetto che indichi il valore unitario delle quote di partecipazione e il valore complessivo dell'organismo di investimento.

48 Recita l'art. 23, comma 6, del testo unico della finanza che "nei giudizi di risarcimento dei danni cagionati al cliente nello svolgimento dei servizi di investimento e di quelli accessori, spetta ai soggetti abilitati l'onere della prova di avere agito con la specifica diligenza richiesta". 
violazione delle norme di comportamento, dall'altro il gestore è tenuto a fornire la prova positiva di avere agito con la diligenza necessaria (CARBONETTI, 1992, p. 68; TOPINI, 1999, p. 700). ${ }^{49}$

L'investitore vanta nei confronti del gestore un credito di valore, come tale rappresentativo di quel "bene" che é il portafogli in gestione.$^{50}$ Ciò premesso, risulta evidente come "[...]'obbligazione di dare danaro costituisca un sostitutivo di un'obbligazione primaria alla quale deve essere equivalente" (QUADRI, 1985, p. 471 ss.)., anche se la pratica sempre più spesso conosce criteri di adeguamento monetario automatico della prestazione che spersonalizzano il lucro cessante, ${ }^{51}$ inteso quale incremento patrimoniale netto che il danneggiato avrebbe ricavato dalla prestazione inadempiuta; ${ }^{52}$ incremento patrimoniale netto che non si é realizzato a causa dell'inadempimento. ${ }^{53}$ Anche il danno prodotto da investimenti non autorizzati dal cliente é un danno risarcibile se e nella misura in cui le operazioni effettuate siano incompatibili con le linee di investimento prescelte. ${ }^{54}$ Emerge dunque la rilevanza concreta, nella quantificazione del danno, delle istruzioni impartite dall'investitore e delle "caratteristiche della gestione" come precisate nel contratto. ${ }^{55}$ L'approccio della giurisprudenza di merito appare meno soddisfacente per l'aspetto della quantificazione del lucro cessante, che spesso é drasticamente legata a parametri monetari: liquidata, cioè, come se si trattasse di risarcire per equivalente un debito pecuniario. Questo accade in primo luogo perché é difficile validare un criterio astratto di determinazione del mancato guadagno, e poi perché si dimentica che il mancato guadagno é rappresentato in questo caso dall'infruttuosa gestione di un portafogli, cioè non di semplice denaro ma di un bene. II portafogli é infatti comunque un bene, per quanto mutevole sia la sua composizione ${ }^{56}$ dato che la sua struttura e consistenza variano in relazione alla vicende gestorie. ${ }^{57}$ II danno risarcibile viene quindi equiparato al danno da inadempimento di un debito pecuniario liquido ed esigibile, ${ }^{58}$ mentre dovrebbe essere equiparato a un debito di valore, che per sua natura é illiquido (ASCARELLI, 1959, p. 450), e deve essere prima stimato, poi liquidato (rectius: reso liquido) e quindi convertito in un'espressione monetaria. La modalità tradizionale di conversione è rappresentata normalmente dall'attribuzione di interessi compensativi, sempre che non sia possibile la reintegrazione in forma specifica. ${ }^{59}$

II limite di questa giurispudenza consiste dunque nel fatto che gli interessi "compensativi" (ossia rappresentativi della stima e della conseguente conversione in moneta di quel debito naturalmente illiquido che è rappresentato dal lucro cessante) sono limitati al mancato guadagno da ritardo (nella liquidazione dei

49 In questo senso anche Trib. Monza, 14 ottobre 2004, in Contr., 2005, p. 113 ss., con commento di GUERINONI, ove il Collegio precisamente afferma che nell'ipotesi di cattivo esito di un'operazione di investimento spetta all'intermediario dimostrare che la negligenza contestata non sussiste.

50 Ascarelli (1959, p. 475), dove l'osservazione, a proposito del debito di valore, che il denaro si intende "[...] come succedaneo di altro bene, ogni qual volta l'obbligazione di dare danaro costituisce un succedaneo, un sostitutivo di un'obbligazione primaria alla quale deve dunque essere equivalente"; Quadri (1985, p. 473), che riassume il concetto di debito di valore come quello "[...] in cui la prestazione è diretta ad integrare o reintegrare il patrimonio del creditore in misura corrispondente ad un determinato valore". Nella giurisprudenza si esprime in termini simili Trib. Biella, 24 gennaio 2001, (nt. 24), 2337.

51 Quadri (1985, p. 474), il quale parla, in proposito, di "crisi" della figura del debito di valore, che, "[...] per il suo carattere individualizzante appare... destinata a cedere il passo a tecniche differenti e per definizione più adatte a risolvere conflitti di rilevanza generale e sociale". In tal modo l'individuazione del (la misura del) lucro cessante passa, essenzialmente, dalle mani del Giudice a quelle del legislatore.

52 Ex pluris v. De Cupis (1939, p. 12), ove la definizione per la quale lo scapito prodotto dall'atto colposo "[...] è costituito dalla differenza ch'è tra lo stato del patrimonio nel tempo in cui la colpa è avvenuta e lo stato in cui era prima, e quello in cui sarebbe stato se alla obbligazione si fosse data la dovuta esecuzione"; (BIANCA, 1979, p. 278).

53 Ai sensi dell'art. 1223 cod. civ., "il risarcimento del danno per l'inadempimento o per il ritardo deve comprendere così la perdita subita dal creditore come il mancato guadagno, in quanto ne siano conseguenza immediata e direta".

54 Non è in sé risarcibile, invece, il danno da "squilibri" interni nella gestione, ossia derivante da una sproporzione tra tipologie di strumenti finanziari prescelti dal gestore professionale, a meno che il contratto non preveda percentuali massime di investimento nelle singole categorie di strumenti finanziari. Si v. in questo senso, infatti, App. Paris, 9 décembre 2004, n. 03/19674, in Bull. Joly Bourse, 2005, 432 ss., con nota di Forgues, Gestion de portefeuille, valeurs TMT et faute du gestionnaire.

55 Sulla disciplina delle "caratteristiche della gestione" v. supra, nt. 38.

56 Il concetto di bene è inteso qui in senso ampio (e v. anche infra, nt. 85). Sul concetto di bene quale sinonimo di "cosa", o res utile, si rinvia ex pluris a Maiorca (1937, p. 10 ss.); Costantino (1982, p. 13); Zeno-Zencovich, (1989, p. 446).

57 V. supra, §1

58 Trib. Monza, 14 ottobre 2004, (nt. 53), p. 115.

59 Bigliazzi Geri-Breccia-Busnelli-Natoli (1993, p. 728) è da precisare che gli interessi compensativi non necessariamente corrispondono agli interessi legali: in questo senso Cass., sez. un., 17 febbraio 1995, n. 1712, in Riv. dir. comm., 1995, II, p. 247, ove l'osservazione che "[...] l'equità potrebbe far ritenere eccessivo un interesse del 10\%, quale è quello attuale". Esiste, tuttavia, una ricca casistica giurisprudenziale dalla quale risulta che è frequente che il lucro cessante sia forfettariamente liquidato nella misura degli interessi legali, ex art. 1224, comma 1, cod. civ.: v. riferimenti in Dellacasa (1999, p. 185, testo e nt. 84). 
valori in dipendenza dal recesso) e non comprendono il mancato guadagno legato ai futuri incrementi che il portafogli avrebbe potuto avere in ragione di una gestione diligente. E' vero che nella quantificazione di quest'ultima voce del lucro cessante non ci si può spingere fino a includere nel risarcimento danni puramente futuri e del tutto incerti, ${ }^{60}$ ma bisogna comunque tenere conto del diritto soggettivo rappresentato dalla prospettiva di un probabile e ragionevole incremento di valore del portafogli. ${ }^{61}$ Anche quest'ultimo deve essere convertito in un credito, che diviene di valuta per effetto della liquidazione giudiziale ${ }^{62}$ Quest'ultima voce di lucro cessante, invece, normalmente manca nei risarcimenti liquidati dal giudice ${ }^{63}$

Bisogna inoltre ricordare che anche qualora non sia possibile il risarcimento in forma specifica, ex art. 2058, comma 10, cod. civ., la liquidazione di interessi compensativi non é l'unica modalità possibile di risarcimento del lucro cessante: ${ }^{64}$ in particolare esiste l'alternativa della liquidazione giudiziale succedanea ad una valutazione equitativa, ex artt. 1226 e 2056, comma 2o, cod. civ. ${ }^{65}$ che presenta anche il pregio di una maggiore elasticità. ${ }^{66}$

Rimane a questo punto da appurare se sia utilizzabile qualche strumento di tutela processuale tecnicamente più soddisfacente e più adatto, ${ }^{67}$ o quanto meno qualche ausilio ulteriore del quale il Giudice possa avvalersi nella valutazione equitativa, ex art. 1226 cod. civ.

\section{Le peculiari modalità di valutazione del danno nel contratto di gestione di portafogli}

La dottrina è sostanzialmente pacifica nel negare da un lato che possa ricomprendersi nel lucro cessante, quale credito di valore illiquido, un vantaggio futuro e incerto, ${ }^{68}$ dall'altro nel negare che il gestore professionale si obblighi per contratto a garantire all'investitore un utile netto determinato, e quindi un vantaggio patrimoniale certo. ${ }^{69}$ Questa conclusione é conforme al fatto che il contratto di gestione di

60 Notoriamente è esclusa la risarcibilità dei danni futuri: cfr. Bigliazzi Geri-Breccia-Busnelli-Natoli (1993, p. 729), osservando non essere risarcibile il danno futuro stricto sensu, "[...] ossia la semplice perdita di quella che la giurisprudenza francese chiama una chance de profit [...]". Si tratta, piuttosto, di risarcire un danno certo nell'an, incerto (solo) nel quantum: Mastropaolo (2003, p. 592), che in proposito parla di lucro cessante come di "[...] eventuale concreta, anche se futura, "redditività perduta" oppure di "occasioni più redditizie perdute" e loro quantificazio- ne pecuniária". In termini analoghi, si osserva che deve essere risarcito il lucro cessante quando, "[...]sulla base della proiezione di situazioni già esistenti, possa ritenersi che il danno si produrrà nel futuro secondo una ragionevole e fondata previsione": (FRANZONI, 1996, p. 430).

61 Del resto, si osserva che "la perdita probabile di un'utilità futura è, sotto un doppio profilo, un'ipotesi da verificare; non è mai una certezza perché, se ci fosse la certezza dell'utilità e della perdita, avremmo un puro e semplice danno emergente. Ora, la probabilità dell'utilità e la probabilità della perdita sono ipotesi da verificare sul piano del grado della probabilità medesima, della consistenza dell'utilità, del tempo del suo possibile verificarsi o mancare". (OPPO, 2000, p. 225).

62 Ascarelli (1959, p. 482), precisando che "la liquidazione giudiziale dei debiti di valore (che debbano essere soddisfatti in danaro) non potrà a sua volta che aver luogo in valuta"; (PINORI, 2001, p. 28).

63 In questo senso infatti Trib. Catania, 5 aprile 2004, cit., che nega il riconoscimento di tale pretesa ritenendo che l'attrice "[...] in sede conclusionale, ha prospettato astratti criteri di determinazione del mancato guadagno a sostegno dell'esistenza di un pregiudizio sofferto diverso ed ulteriore rispetto a quello verificatosi al momento del recesso dal contrato". Bisogna notare che questa parte della motivazione è integralmente tratta dalla motivazione di Trib. Biella, 24 gennaio 2001, (nt. 24), 2338, che ugualmente negava il riconoscimento, e la conseguente liquidazione, di voci di lucro cessante ulteriori rispetto agli interessi legali in quanto "[...] dagli atti non si ricavano elementi concreti dai quali desumere, in pendenza di mandato gestorio, la condotta alternativa lecita che in quel preciso contesto economico-finanziario parte convenuta avrebbe potuto adottare invece di acquistare i titoli nella specie contestati per ottenere dal complesso dell'attività espletata, di cui le negoziazioni in questione costituiscono solo una parte dell'adempimento, un certo utile determinato". Sulle modalità di determinazione del lucro cessante v. anche Trib. Monza, 14 ottobre 2004, (nt. 53), loc. ult. cit., ove il Collegio rinvia ai numerosi precedenti conformi. In particolare tra questi, entro un ricco filone di pronunce in materia di liquidazione dei debiti di valore, Cass., sez. un., 17 febbraio 1995, n. 1712, (nt. 65), 239 ss.

64 Cfr. Cass., sez. un., 17 febbraio 1995, n. 1712, (nt. 65), 247, ove l'osservazione che "[...] l'attribuzione degli interessi quale lucro cessante, costituisce solo una modalità di liquidazione equitativa (salvo prova diversa) e non un'obbligazione accessoria di un'obbligazione di valore".

65 Dispone l'art. 1226 che "se il danno non può essere provato nel suo preciso ammontare, è liquidato dal giudice con valutazione equitativa"; dispone l'art. 2056, comma 2o, che "il lucro cessante è valutato dal giudice con equo apprezzamento delle circostanze del caso".

66 Ex pluris Bigliazzi Geri-Breccia-Busnelli-Natoli (1993, p. 728 ss.); Lobuono (2002, p. 265); Salvatore (2004, p. 407).

67 In argomento si v. le considerazioni di Taruffo (1999, p. 788 ss.), che rileva come le controversie ad alto contenuto tecnico-scientifico, tra le quali si ascrivono certamente quelle relative ai mercati finanziari nazionali e internazionali, richiederebbero modalità più efficaci della consulenza tecnica d'ufficio, che appare per molti versi inadeguata, così come richiederebbero, con ogni probabilità, una competenza specialistica dello stesso organo giudicante. A ciò si aggiunga che sul piano dei rimedi l'inibitoria può probabilmente rivelarsi strumento più utile a tutelare i diritti che sorgono sui mercati, ben più del risarcimento danni. Di fronte ai limiti delle inibitorie tipiche l'Autore ipotizza, piuttosto, che al collegio giudicante potrebbe essere attribuito un "potere inibitorio generale", "...da esercitare a seconda delle circostanze e con la possibilità più ampia di adattare il provvedimento a tali circostanze". Favorevole ad una valorizzazione dell'uso dei rimedi di tipo inibitorio nelle controversie relative ad operazioni di investimento anche Topini (nt. 16, p. 733).

68 V. supra, fine $\S 3$.

69 Si v. Lobuono (1999, p. 269 ss.); Mastropaolo (2003, p. 585 ss.); cfr. Gaeta (2002, nota a Trib. Mantova, 12 novembre 2004 , p. 590$)$. 
portafogli é fonte per il gestore di una obbligazione di mezzi e non di risultato, come si é già detto, ${ }^{70} \mathrm{e}$ al fatto che l'autorità giudiziaria non può censurare nel merito l'attività gestoria, né i singoli atti in cui si estrinseca. ${ }^{71} \mathrm{Vi}$ è infatti una intrinseca alea economica nel tipo contrattuale, che nemmeno con l'ausilio del più elevato livello di perizia può essere neutralizzata. ${ }^{72}$ Piuttosto, può correttamente dirsi che obiettivo dell'investitore è ottenere, tramite l'operatore professionale, una gestione utile nel senso che il pensiero filosofico utilitaristico attribuisce al termine. ${ }^{73}$

Ciò premesso, questa innata e parziale imprevedibilità dell'esito della gestione ${ }^{74}$ non può però diventare alibi per il compimento di operazioni di puro rischio, né per la contravvenzione delle istruzioni eventualmente impartite dall'investitore. ${ }^{75}$

Sul piano poi delle tecniche probatorie, sono state trascurate finora le potenzialità del benchmark, o parametro oggettivo di riferimento, che pure è assurto al rango di criterio normativo, giusto l'esplicito riferimento al medesimo contenuto nell'art. 29, par. 2, del regolamento intermediari. ${ }^{76}$ La costruzione di un benchmark mira alla rappresentazione di un portafogli economicamente efficiente, ossia tale che relativamente, e limitatamente, al mercato cui il benchmark stesso si riferisce "[...] offra il migliore tradeoff rendimento rischio", ${ }^{77}$ salva la difficoltà scaturente dal fatto che non esiste un unico giusto modello di determinazione del rischio (ASSOGESTIONI, 2002, p. 22).

Nella pratica accade invece che la richiesta di liquidazione di danni da lucro cessante non trovi accoglimento. Non sempre si indaga a sufficienza negli atti di causa per verificare se ci siano elementi bastanti a fondare un giudizio di probabilità sull'evoluzione prevedibile che la gestione di portafogli avrebbe dovuto avere se l'impresa di investimento avesse tenuto un comportamento diligente. Ed é arbitrario (de)limitare la liquidazione del danno da lucro cessante al ritardo nell'accredito dei valori disinvestiti in pendenza del recesso, posto che il recesso rappresenta soltanto la reazione postuma al "fallimento» del progetto gestorio. E nella prospettiva eziologica della gestione di portafogli la mera restituzione di un valore monetario non costituisce una adeguata riparazione al danno, perché il bene in questo caso non è rappresentato da dalla somma che é stata inizialmente affidata al gestore, ma un portafogli utilmente gestito e diversificato, che quale che sia la conformazione che via via esso assuma, nella fase esecutiva del contratto, in ragione delle scelte di investimento operate dal gestore professionale, deve costantemente essere oggetto di considerazione e tutela unitaria. ${ }^{78}$ Sulla base delle considerazioni svolte, dunque, risulta che al di là dell'obiettiva difficoltà di prevedere con ragionevole certezza quale potrà essere la performance

\footnotetext{
V. supra, inizio § 2

71 Si v. Topini (1999, p. 722 s.): evidentemente, "[...] è opportuno che i giudici sanzionino unicamente le operazioni palesemente irrazionali e contrarie ai principi di sana e prudente gestione, quelle avvenute in conflitto di interesse con il cliente, quelle ancora che richiedono impegni economici del cliente sproporzionati rispetto al patrimonio affidato in gestione, quelle infine particolarmente rischiose".

72 Sulla nozione e la reciproca distinzione (talvolta labile) di alea giuridica e alea economica v. Scalfi (1960, p. 111 ss.), ove si ripercorrono le tappe della dottrina che ha delimitato il contratto aleatorio in senso stretto ravvisando il connotato tipico della categoria "[...] nell'incidenza dell'evento incerto sull'esistenza o sulla determinazione di una prestazione". Più recentemente cfr. Gabrielli (2001, p. 1 ss.); Corgill (1993, p. 875), ove la constatazione che "[...] risk includes the chance of receiving more than the most anticipated outcome or, for that matter, more than the outcome that was thought, ex ante, to be the most favorable outcome. An investment at economic risk, then, means that the investor might receive more or less than the expected value".

73 Così Di Maio (1994, p. 323), con evidente riferimento all'utilitarismo benthamiano. Osserva, dunque, l'A. che la causa del contratto include una gestione profittevole, eventualmente anche in perdita se la perdita è comunque minore di quella che si sarebbe sofferta senza l'ausilio di un gestore professionale.

74 Osserva ancora Corgill (1993, p. 876), che "[...] the actual financial reward is a random event because it is impossibile to predict the precise financial reward that an investor will receive for a particular investment...Some deviations are uncertain because they result from market forces or other processes that defy prediction... Where deviations are caused by this type of uncertainty, it is impossible to predict the likelihood of any particular outcome or deviation from the expected value".

75 Si v. Mastropaolo, (2003, p. 610); cfr. Cass., 15 gennaio 2000, n. 426, (nt. 27), 919, ove il s.C. incisivamente precisava, in materia di mandato alla gestione di azioni, che "non è sufficiente contrapporre l'aleatorietà e la rischiosità delle operazioni di borsa, sia perché ciò non si identifica con la "rovinosità" ed il puro azzardo, sia soprattutto perché tali caratteristiche non giustificano gli elevati costi incontrati nella gestione [...]".

76 V. supra, § 2 e nt. 38.

77 Assogestioni (2000, p. 35; v. anche, ivi, 21 ss.) con riferimento al concetto di covarianza tra rendimento dell'attività e rendimento del portafoglio di riferimento, e alla teoria di portafoglio tradizionale detta Capital Asset Pricing Model (CAPM), che sostanzialmente identifica il beta del rendimento di un certo titolo con il rendimento del portafoglio di mercato quale indicatore di rischio; (COSSU, 2011, p. 207 ss.).

78 Questo aspetto della problematica é legato alla questione della natura del portafogli in gestione, e alle connesse difficoltà di suo inquadramento sistematico all'interno della teoria generale dei beni. In argomento v. Lobuono (2002, p. 48 ss.), ove più generali riferimenti alle new properties.
} 
degli strumenti finanziari, e del fatto che non è possibile eliminare l'alea economica degli stessi, e fermo restando che anche l'eventuale riferimento ad un paniere di "beni" aventi caratteristiche analoghe in termini di rischio, rendimento, duration non consente di simulare perfettamente quella che avrebbe potuto essere l'evoluzione reale dell'investimento, la conclusione é che la tecnica processuale ancora non sfrutta appieno le potenzialità del benchmark; ${ }^{79}$ con la conseguenza che la valutazione del danno nella gestione di portafogli finisce per appiattirsi nell'alveo della valutazione del danno da inadempimento dell'obbligazione pecuniaria. $^{80}$

Al fine di determinare e liquidare la componente lucro cessante nei giudizi di risarcimento danni da gestione negligente dovrebbe, dunque, essere implementato l'utilizzo del benchmark sia in funzione ancillare rispetto alla valutazione giudiziale equitativa, ex art. $1226 \mathrm{cod}$. civ. ${ }^{81}$ e sia anche, e forse meglio, quale strumento autonomo.

\section{Riferenze}

ALCARO, Francesco. La categoria dell'attività: profili ricostruttivi. (Atti e attività. L'attività d'impresa). Riv. crit. dir. priv., Napoli, v. 13, n. 2, p. 417 ss.1995.

ALCARO, Francesco. Mandato e attività professionale. Milano: Giuffré, 1988.

ALPA, Guido; CAPRIGLIONE, Francesco. Art. 23. Contratti. In: ALPA, Guido; CAPRIGLIONE, Francesco (Coord.). Commentario al testo unico delle disposizioni in materia di intermediazione finanziaria. Padova: Cedam, 1998.

AMOROSINO, Sandro. Manuale di diritto del mercato finanziario. Milano: Giuffré, 2014.

ANNUNZIATA, Filippo. La disciplina del mercato mobiliare. Torino: Giappichelli, 2015.

ARU, Luigi. Art. 2028-2042. Della gestione di affari. In: Commentario Scialoja-Branca (nt. 10), IV, Delle obbligazioni. Bologna-Roma: Zanichelli, p. 674-1343, 1957.

ASCARELLI, T. Delle Obbligazioni pecuniarie. In: Commentario del Cod. Civ. Scjaloia- Branca, (nt. 10), Bologna - Roma: 1959.

ASSOGESTIONI. Benchmark e fondi comuni. Milano, 2000.

BAVETTA, Giuseppe. voce Mandato (dir. priv.). Enc. dir., v. XXV, Milano: Giuffré, 1975.

BIANCA, Cesare Massimo. Dell'inadempimento: Delle obbligazioni: Art. 1218-1229. In: Scialoja-Branca

(Coord.). Commentario de Codice Civile. (nt. 10), libro IV, 2 ed., Bologna-Roma,1979.

BIANCA. Vincoli di destinazione e patrimoni separati. Milano: CEDAM, 1996.

BIGLIAZZI, Geri; BRECCIA, Umberto; BUSNELLI, Francesco. Diritto civile: obbligazioni e contratti. Torino: Utet, 1993. v. 3.

79 Su questa linea Lobuono (1999, p. 271), dove l'osservazione che, all'insegna di un benchmark, "[...] il danno di cui il cliente può chiedere il risarcimento a titolo di mancato guadagno dovrebbe essere calcolato avendo riguardo al...valore medio dei rendimenti conseguiti dai prodotti finanziari facenti parte del paniere di riferimento, nel periodo corrispondente a quello in cui le parti sono state legate dal contratto di investimento che ha dato origine all'azione di responsabilità".

80 Una parziale evoluzione del trend giurisprudenziale può vedersi in Trib. Lecce, 28 giugno 2004, in questa Rivista, 2005, II, 584, dove il quantum risarcibile viene individuato "[...] in ragione dei risultati che il danneggiato avrebbe conseguito dalla puntuale negoziazione dei titoli". La consulenza tecnica d'ufficio, pertanto, viene utilizzata per individuare il rendimento auspicabile dell'investimento.

81 Questa eventualità è prospettata da Morozzo Della Rocca (1991, p. 899), ove l'A. osserva che nella valutazione equitativa ex art. 1226 c.c. "[...] il riferimento all'andamento medio dei rendimenti potrà costituire un valido criterio a disposizione dei giudici". E in merito all'utilizzo del benchmark quale parametro di riferimento al quale rapportare l'andamento della gestione v. in particolare Cass. civ., 21 aprile 2016 , n. 8089, "Gas Rimini s.p.a." c. "Fideuram s.p.a.", in Dir. bancario.it, aprile 2016. Ivi la suprema Corte sancisce il principio secondo il quale il contratto di gestione di portafogli deve preventivamente precisare il grado di rischio di ciascuna linea di gestione patrimoniale, essendo tale prescrizione, prevista nell'Allegato 3 sub C) del Regolamento intermediari, vincolante, e dettata al fine di indicare le modalità di esecuzione dell'obbligo, sancito nell'art. 42 del medesimo regolamento, di fornire all'investitore un parametro oggettivo coerente dei rischi connessi alle singole gestioni. E vedi anche Trib. Biella, 5 aprile 2007, in Banca, borsa, tit. cred. (2009, II, p. 63 ss.), con nota di P. Lucantoni, La responsabilità contrattuale dell'intermediario per la gestione del portafoglio di investimento in difformità al benchmark indicato dal cliente: l'impatto del recepimento della direttiva Mifid, relativamente alla valutazione dell'inadempimento, e conseguente responsabilità, di un gestore di portafogli che aveva agito in difformità al benchmark indicato nel contratto. 
BIONDI, I beni de Biondo. Trattato di diritto civile italiano: diretto da Filippo Vassalli. Torino: UTET, 1956. v. IV, t. I.

BOCHICCHIO, Francesco. Gli strumenti finanziari nella teoria dei titoli di massa: continuità o rottura. II Diritto fallimentare, Milano, v. 72, n. 5, p. 1068-1103, 1997.

BRECCIA, Umberto. La gestione di affari. In: Trattato di diritto privato diretto da Rescigno. Torino: UTET, 1982. v. 9, t. I.

BRECCIA, Umberto. Le obbligazioni. in Trattato di diritto privato, a cura di ludica e Zatti. Milano: Giuffré, 1991.

CARBONETTI, Francesco. I contratti di intermediazione mobiliare. Milano: Giuffré, 1992.

CERA, Mario. Lo statuto speciale delle società di intermediazione mobiliare nel d. Igs. 23 luglio 96,

Banca, borsa, tit. Credito, Milano, v. I, n. 415, p. 719 ss., 1997.

CLEMENT, Jean-François. Le banquier, vecteur d'informations. Riv. trim. droit comm., Paris, n. 15, p. 203 ss., 1997.

CORGILL, Dennis S. Securities as investments at risk. Tulane Law Rev., v. 67, n. 4, p. 3-38, 1993.

COSSU, Monica. Contratti di gestione di portafogli di investimento. Trattato Rescigno-Gabrielli, I contratti del mercato finanziario, diretto da. Gabrielli e Lener, Torino: Utet, 2011.

CossU, Monica. Contratti di investimento. Enc. giur., X, Roma: Treccani, v. 59, n. 1, p. 67-82, 2002-A.

COSSU, Monica. I "patrimoni di destinazione” nella liquidazione coatta amministrativa: la (parziale) svolta della giurisprudenza di merito, nota a App. Bologna, 14 gennaio 2002 e Trib. Catania, 7 marzo 2002, in Banca, borsa, tit. Credito, Bologna, v. 55, n. 3, p. 301-318, 2003.

COSSU, Monica. La "gestione di portafogli di investimento" tra diritto dei contratti e diritto dei mercati finanziari. Milano: Giuffré, 2002-B.

COSTANTINO, Michele. I beni in generale. Trattato di diritto privato diretto da Rescigno, 7, Proprietà, Torino: Utet, 1982. t. I.

COSTI, Renzo; ENRIQUES, Lucas. II mercato mobiliare. In: Trattato Cottino, VIII, Padova: Cedam, 2004. p. 373 ss.

DE CUPIS, Adriano. Contributi alla teoria del risarcimento del danno. Milano: Giuffré, 1939.

DE CUPIS, Adriano. II danno. Teoria generale della responsabilità civile. Milano: Giuffré, 1946.

DELLACASA, Marco. I danni nelle obbligazioni pecuniarie. In: Risarcimento del danno contrattuale ed extracontrattuale, a cura di Visintini. Milano: Giuffré, 1999.

DI MAIO, Federico. Gestione di patrimoni tra società fiduciaria (non iscritta) e sim. Le Società, v. 13, n. 3 , p. 322-325, 1994.

ESSOMBÈ MOUSSIO, Jean-Jacques. La gestion de portefeuille. In: La modernisation des activités financières, sous la direction de BONNEAU. Paris: GLN Joly, 1996.

FAZIO, Eugenio. Dalla forma alle forme. Struttura e funzione del neoformalismo negoziale. Milano: Giuffré, 2011.

$\mathrm{FICl}$, Antonio. Ancora sull'inibitoria ordinaria delle condizioni generali utilizzate dalle banche; con specifico riferimento all'applicabilità dell'art. 1469-bis c.c. v. Trib. Messina, 17 giugno 2000 (ord.). Vita not., Roma, v. 37, n. 2, p. 478-482, 2004.

FRANZONI, Massimo. II danno al patrimônio. Milano: Giuffré, 1996.

GABRIELLI, Enrico. voce Alea. Enc. giur., IX, Roma: Treccani, agg. 2001.

GABRIELLI, Enrico; ORESTANO, Andrea. voce Contratti del consumatore. Digesto disc. priv., sez. civ., IV, Torino: Utet, 2000 
GAETA, M. M. La gestione individuale dei portafogli di investimento. La discrezionalità del gestore e l'autorizzazione del cliente. Giur. Comm., Milano, n. 87, p. 113 ss., 2002.

GIOIA, Gina. Tutela giurisdizionale dei contratti del mercato finanziario. Trattato Rescigno-Gabrielli, I contratti del mercato finanziario, diretto da. Gabrielli e Lener. Torino: Utet, 2011.

GUERRERA, F. Contratto d'investimento e rapporto di gestione. (Riflessioni sulla proprietà delegata). Riv. crit. dir. priv., Roma, v. 3, n. 77, p. 698 ss., 1988.

LARENZ, Karl. Lehrbuch des Schuldrechts, II, Besonderer Teil, München, Beck, 1972.

LENER, Raffaele. Forma contrattuale e tutela del contraente "non qualificato" nel mercato finanziario. Milano: Giuffré, 1996.

LENER, Raffaele. voce Valori mobiliari, II), Soggetti intermediari. Enc. giur., XXXII, Roma: Treccani, 1994.

LOBUONO, Michele. I "nuovi beni" del mercato finanziario. Riv. dir. priv., ano 114, n. 22002.

LOBUONO, Michele. La responsabilità degli intermediari finanziari. Napoli: Esi, 1999.

LUCANTONI, Paola. Le regole di condotta degli intermediari finanziari. Trattato dei contratti, diretto da P. Rescigno e E. Gabrielli, I contratti del mercato finanziario, a cura di E. Gabrielli e R. Lener. Torino: Utet, 2011, p. 239-278.

LUMINOSO, Angelo. II mandato e la comissione. Trattato di diritto privato diretto da Rescigno, 12, Obbligazioni e contratti. Torino: Utet, 1985.

MAGGIOLO, Marcello. II risarcimento della pura perdita patrimoniale. Milano: Giuffré, 2003.

MAGNO, Daniele Magno Premessa. Mala gestio e profili di responsabilità del gestore patrimoniale. In: Trattato Rescigno-Gabrielli, I contratti del mercato finanziario, diretto da. Gabrielli e Lener. Torino: Utet, 2011.

MAIORCA, Carlo. La cosa in senso giuridico. Napoli: Esi, 1937.

MASTROPAOLO, Eugenio M. I servizi di investimento e gli intermediari professionali. Milano: Giuffré, 2003.

MENGONI, Luigi. Obbligazioni "di risultato" e "obbligazioni di mezzi”. (Studio critico). Riv. dir. comm., Milano, ano 50, n. 1, p. 368-371, 1954.

MOROZZO DELLA ROCCA, Paolo. Appunti sulla responsabilità dell'intermediario nel rapporto di gestione personalizzata di portafogli. Riv. crit. dir. priv., Roma, p. 865-899, 1991.

OPPO, Giorgio. Scritti giuridici. v. 6: Principi e problemi del diritto privato. Padova: Cedam, 2000.

OPPO, Giorgio. Sui principi generali del diritto privato. Riv. dir. civ., Milano, v. 1, p. 475, 1991.

PICARDI, Lucia. Impresa e contratto nella gestione del risparmio. Milano: Giuffré, 2004.

PINO, Augusto. II patrimonio separato. Padova: Cedam, 1950.

PINORI, Alessandra. II danno contrattuale. Padova: Cedam, 2001.

QUADRI, Enrico. Le obbligazioni pecuniarie. Trattato Rescigno, (nt. 24). Torino: Utet, 1985. v. 9. t. I.

RODOTÀ, Stefano. II tempo delle clausole generali. Riv. crit. dir. priv., Milano, v. 1, p. 709-733, 1987.

RONDINONE, Nicola. L' "attività" nel codice civile. Milano: Giuffré, 2001.

ROPPO, Vincenzo. Contratto di diritto comune, contratto del consumatore, contratto con asimmetria del potere contrattuale: genesi e sviluppi di un nuovo paradigma. Riv. dir. priv., Torino, v. 1, 2001.

ROVITO, V.; PICARDI, L. Articolo 23. Contratti. In: Testo Unico della finanza. Commentario, a cura di G.F. Campobasso. Intermediari e mercati. Torino: Utet, 2002.

SALANITRO, Nicola. Società per azioni e mercati finanziari. Milano: Giuffré, 1998. 
SALVATORE. Michelangela. Servizi di investimento e responsabilità civile. Milano: Giuffré, 2004.

SANTORO PASSARELLI, Francesco. Dottrine generali del diritto civile. Napoli: Jovene, 1968.

SARTORI. Le regole di adeguatezza e i contratti di borsa: tecniche normative, tutele e prospettive MiFID. Riv. dir. priv., Milano, v. 3, 2008.

SCALFI, Gianguido. Corrispettività e alea nei contratti. Napoli: Esi, 1960.

TARUFFO, Michele. Adeguamenti delle tecniche di composizione dei conflitti di interesse. Riv. trim. dir. e proc. civ., Roma, v. 53, n. 3, p. 779-792, 1999.

TOPINI, M. L'onere della prova nei giudizi di responsabilità per danni cagionati nello svolgimento dei servizi di investimento. Giur. Comm., Milano, n. 6, p. 697-713, 1999.

VENUTI, M. C. Le clausole generali di correttezza, diligenza e trasparenza nel Testo Unico delle disposizioni in materia di intermediazione finanziaria. Europa e dir. priv., Milano, 2000.

VISINTINI-CABELLA PISU, Luciana. L'inadempimento delle obbligazioni. Trattato di diritto privato, diretto da Rescigno, 9, Obbligazioni e contratti. Torino: Utet, 1986. t. I.

ZENO-ZENCOVICH, Vicenza. voce Cosa. Digesto disc. priv, sez. civ. Torino: Utet, v. 3, 1989. t. I.

Recebido em : 09/08/2017

Aprovado em : 20/09/2017 